\title{
Efectos de la responsabilidad social empresarial sobre la identificación organizacional de los empleados: autenticidad o falacia
}

\author{
Effects of corporate social responsibility on \\ employee organizational identification: Authenticity or fallacy \\ Orlando E. Contreras-Pacheco ${ }^{1 *}$, Leonardo H. Talero-Sarmiento ${ }^{2}$, \\ Julio C. Camacho-Pinto ${ }^{2}$ \\ ${ }^{1}$ Universidad Industrial de Santander, Colombia - Rennes School of Business, Francia \\ ${ }^{2}$ Universidad Industrial de Santander, Colombia
}

Recibido el 23 de agosto de 2017; aceptado el 1 de febrero de 2017

Disponible en Internet el: 20 de junio de 2019

\section{Resumen}

El greenwashing implica el predominio del uso falaz de la responsabilidad social empresarial (RSE simbólica) sobre la práctica auténtica de la misma (RSE sustancial). Su definición representa una tensión conceptual, susceptible de ser estudiada a la luz de su impacto sobre la identificación de los individuos como miembros de una organización. El presente trabajo estudia dicho impacto por medio de una investigación transversal aplicada sobre doscientos veinte empleados profesionales en Colombia y analizada a través del modelamiento con ecuaciones estructurales. Los resultados obtenidos dan cuenta del carácter asociativo de la RSE sustancial sobre la identificación organizacional de los empleados, así como de la ausencia de vínculo entre la RSE simbólica sobre esta misma actitud. Sin embargo, también encuentra una correlación directa entre la RSE simbólica y la RSE sustancial que podría suponer una aceptación

\footnotetext{
${ }^{*}$ Autor para correspondencia

Correo electrónico ocontrer@uis.edu.co (O.E. Contreras-Pacheco)

La revisión por pares es responsabilidad de la Universidad Nacional Autónoma de México.

http://dx.doi.org/10.22201/fca.24488410e.2018.1631

0186- 1042/C 2019 Universidad Nacional Autónoma de México, Facultad de Contaduría y Administración. Este es un artículo

Open Access bajo la licencia CC BY-NC-SA (https://creativecommons.org/licenses/by-nc-sa/4.0/)
} 
tácita del greenwashing como una práctica válida. A nivel teórico, se contribuye en la medida en que se prueba por primera vez un modelo conceptual usando constructos de reciente incorporación a la literatura. Asimismo, a nivel práctico, se sugiere que la toma de decisiones sobre aspectos relacionados con la RSE está en continuo escrutinio por parte de los empleados de acuerdo a la autenticidad de su adopción y con posibles consecuencias sobre sus comportamientos.

Código JEL: M10, M12, M14

Palabras clave: Greenwashing; Identificación organizacional; Legitimidad; Micro RSE

\begin{abstract}
Greenwashing implies the predominance of the fallacious application of the corporate social responsibility (Symbolic CSR) over its authentic practice (Substantive CSR). It represents, by definition, a conceptual conflict, worth it to observe in light of its impact on the organizational identification of employees. This work examines this impact by carrying out a cross-sectional investigation and analyzing it through structural equation modeling. The study is applied to a sample of two hundred and twenty professional employees in Colombia. Results obtained are mainly in line with the theory; they show a positive effect of Substantive CSR on employees' organizational identification, as well as a futile character of Symbolic CSR on this same attitude. However, the study also finds a direct correlation between Symbolic CSR and Substantive CSR, which suggests a possible tacit acceptance of greenwashing as a valid organizational practice. This work contributes to enhance the theory, given the fact that a recently proposed conceptual model was academically tested for the first time ever. Likewise, at a practical level, results suggested that the decision-making process when related with CSR is under continuous scrutiny by employees, in function of its sense of authenticity and therefore with consequences that may affect their behavior.
\end{abstract}

JEL code: M10, M12, M14

Keywords: Greenwashing; Legitimacy; Micro CSR; Organizational identification

\title{
Introducción
}

El greenwashing, como idea antagónica del sentido auténtico de la responsabilidad social empresarial (RSE), se ha erigido como un tópico emergente cuyos medios y fines han cautivado a académicos y profesionales que buscan entender su funcionamiento y al mismo tiempo mitigar su propagación (Lyon \& Montgomery, 2015). Una de las múltiples maneras de hacerlo podría ser a través del análisis del efecto generado por su adopción sobre los empleados y sus actitudes en el medio laboral (Donia \& Tretault-Sirsly, 2016). Particularmente, la identificación 
organizacional (en inglés: organizational identification u OID, como se referenciará de ahora en adelante) se ha consolidado en la literatura como un constructo que permite la conexión entre una serie de estímulos organizacionales con algunos resultados comportamentales en el plano individual (Lee, Park \& Koo, 2015). Por tanto, teniendo en cuenta que esta actitud ha sido ampliamente analizada a la luz de diferentes manifestaciones a nivel organizacional, se puede pensar que también sería pertinente evaluar la forma en que ella podría ser afectada por la adopción (o no) de prácticas coincidentes con el greenwashing; lo cual sería determinada por la percepción sobre la autenticidad (o no) de la RSE.

Es así como por una parte se tiene claro que la concepción teórica del greenwashing, interpretado como un ejercicio de engaño, puede ser un efectivo antecedente de efectos negativos tanto para las organizaciones como para las sociedades en general (Lyon \& Montgomery, 2015). Y por la otra, se advierte que algunos resultados organizacionales como lo son un mayor compromiso y motivación, menor ausentismo y mayor rendimiento en el puesto de trabajo son a su vez impulsados por adecuados niveles de OID en los empleados (Jones \& Volpe, 2011). No obstante, aún se requiere un mayor y más refinado conocimiento sobre la conexión que pueda existir entre dichos conceptos (Glavas \& Godwin, 2013). Especialmente en contextos particulares en donde aspectos como el impacto medio-ambiental y social generado por la dinámica de las organizaciones es crítico para la construcción del desarrollo futuro de sus sociedades (Jamali \& Karam, 2016; Rodrigo, Duran \& Arenas, 2016

De esta manera, entendiendo al greenwashing como la diferencia entre las acciones falaces y auténticas relacionadas con la RSE (Walker \& Wan, 2012), y a la OID como la autopercepción de un empleado expresada como una aproximación a su propio sentido de pertenencia hacia la organización de la cual es miembro (Jones \& Volpe, 2011), el presente trabajo se pregunta por el impacto que lo primero puede ejercer sobre lo segundo. En otras palabras, se pretende identificar qué tanto influye la naturaleza auténtica o falaz de la RSE de una organización sobre el sentido de pertenencia de sus empleados hacia la misma. En ese orden de ideas, el marco de trabajo utilizado integra conceptualmente algunos aportes de la teoría de la legitimidad organizacional (representada por la noción de greenwashing) con la fundamentación en RSE a nivel individual, o micro RSE (representada por la OID). En lo metodológico, se lleva a cabo una investigación transversal exploratoria que busca evaluar empíricamente un modelo teórico en donde la OID adquiere el rol de variable dependiente y se interrelaciona con la RSE analizada desde sus percepciones sustancial (práctica y auténtica) y simbólica (retórica o falaz). Específicamente, se acude a un estudio multivariante que abarca una muestra de doscientos veinte empleados profesionales con cargos de responsabilidad en Colombia. De esta forma, tres hipótesis fueron puestas a prueba mediante un modelo de 
ecuaciones estructurales fundamentado en análisis factoriales exploratorios y confirmatorios. La estructura del manuscrito se compone de cinco capítulos adicionales. En primera instancia se presenta un marco teórico en el que se exponen los constructos a analizar en conjunto con antecedentes científicos donde se evidencia de una manera más profunda la pertinencia de su asociación teórica. Posteriormente vendrá la exposición de la metodología empleada, en donde se desarrollan las hipótesis de trabajo, se detalla el diseño del instrumento y se formula el respectivo modelo de ecuación estructural. En el cuarto capítulo se registran los resultados obtenidos en sus diversas etapas. En el quinto capítulo se consigna la discusión generada a partir de la respectiva interpretación de los resultados, sus implicaciones y posibles líneas de investigación futuras. Finalmente, se despliega la conclusión a la que se llega con el estudio.

\section{Marco teórico}

Dentro del cuerpo de conocimiento de la teoría organizacional ha existido un avance interesante con respecto a los efectos generados por las diferentes formas en que la RSE y sus múltiples conceptos asociados impactan a los empleados a nivel de sus percepciones y comportamientos (como la motivación, compromiso, satisfacción, intención de renuncia e identificación, entre otros). A este campo de estudio se le ha denominado Micro Responsabilidad Social Empresarial (Micro RSE), la cual es una derivación del campo de estudio del comportamiento organizacional a nivel del individuo (Rupp \& Mallory, 2015). De la misma forma, la legitimidad organizacional ha jugado un papel importante dentro de esta disciplina, y a pesar de una serie de aportes interesantes que dan luces a esta relación, se advierte continuamente de la existencia de un sinnúmero de oportunidades de la creación de más y mejor conocimiento al respecto (Aguinis \& Glavas, 2012; Delmas \& Pekovic, 2013; Evans \& Davis, 2014; Evans, Davis \& Frink, 2011; Lamm, Tosti-Kharas \& King, 2015). El siguiente marco teórico se estructura de lo general a lo particular, comenzando con la RSE como macro-campo de estudio vinculada al concepto de la legitimidad y finalizando con algunos antecedentes investigativos que de alguna manera las han involucrado con la OID. Finalmente se señalará la brecha específica de la literatura que se pretende abordar con este trabajo.

\section{Responsabilidad social empresarial (RSE)}

Desde que los encargados de la toma de decisiones en las organizaciones entendieron que los objetivos finales no deben ser exclusivamente financieros, sino también sociales y ambientales (Elkington, 1998), el concepto de RSE (también entendido como Responsabilidad Social 
Corporativa o RSC) ha estado muy ligado a los esfuerzos de buscar ese tipo de resultados en sus entornos. En otras palabras, el denominado desarrollo sostenible ha ganado un importante espacio en la gestión (tanto práctica como teórica); implicando que el surgimiento de la RSE, se ha hecho un tema muy relevante en la gestión (Aguilera, Rupp, Williams \& Ganapathi, 2007). El desarrollo sostenible es universalmente definido como "aquel que satisface las necesidades del presente sin comprometer la capacidad de las generaciones futuras para satisfacer sus propias necesidades" (United Nations World Commission on Environment and Development, 1987, p. 3), y de hecho, tal es la influencia de este concepto que incluso ha sido catalogado por algunos autores como el nuevo paradigma dominante en términos de evaluar el desempeño organizacional y personal (Boudreau \& Ramstad, 2005).

La investigación en el campo de la RSE ha mostrado una reciente tendencia hacia la evaluación de la autenticidad en su adopción por parte de las organizaciones (De Roeck \& Maon, 2016). En este sentido, la definición genuina de RSE excluye explícitamente de sus dominios aspectos como el cumplimiento de la ley (incluso de la misma legislación ambiental o laboral), el pago de impuestos y regalías, el intento de compensar una acción irresponsable con otras acciones benévolas (de tipo quid pro quo), el ocultamiento o manipulación de la verdad, las conspiraciones de sectores específicos para incrementar ganancias a costa de sus clientes y proveedores, y la participación directa e indirecta en industrias de explotación indiscriminada de recursos naturales (Berrone, 2016).

En una reciente revisión literaria sobre la psicología organizacional vinculada a la RSE, los profesores Jones \& Rupp (2017) sostienen que la responsabilidad social ha sido comúnmente relacionada con sentimientos, expresiones y acciones. Por lo tanto, "la RSE es a menudo conceptualizada, descrita y ejecutada como un conjunto de prácticas, políticas e iniciativas socialmente orientadas" (ídem; p. 4). Al mismo tiempo, es necesario destacar que la RSE tiene un fuerte vínculo con la teoría de los stakeholders (Freeman \& McVea, 2006), que a su vez se centra en las acciones tomadas en consideración de "aquellos que podrían afectarse por, o afectar el logro de los objetivos de la organización" (ídem; p. 46). En esa misma línea, dado que los empleados, efectivamente representan a un grupo de interesados que pueden afectar y/o se ven afectados por el logro de los objetivos de las organizaciones a las que pertenecen, deben indudablemente ser considerados como stakeholders internos.

\section{Legitimidad y greenwashing}

Las bases teóricas del concepto de legitimidad se remontan a fuertes raíces filosóficas que llegan a temáticas más ancladas en la teoría institucional. Su despliegue va desde el siglo IV 
A.C., con la definición del famoso "acto moral" (good act) de Aristóteles (Koehn, 1995) hasta la exposición de tensiones entre la filantropía y la creación de valor para los accionistas de Godfrey (2005) pasando por el análisis sobre la disociación (o mejor conocida por su término original: decoupling, por su difícil polisemia) entre las estructuras organizacionales reales y aquellas que se desean presentar ante la sociedad (Meyer \& Rowan, 1977). En este sentido, dicha presión por legitimar una imagen responsable puede llevar a las empresas a adoptar prácticas de RSE solo con el fin de cumplir con un formalismo determinado y terminan manipulando la verdad (Ramus \& Montiel, 2005). De esta forma, el greenwashing a nivel de la organización implica efectivamente una disociación entre el compromiso real de la RSE y la presión (legal, social o económica) por la aplicación de RSE. Y tal vez es por ello también que el greenwashing sea un fenómeno que esté continuamente asociado con la divulgación y el manejo de información intencionalmente manipulada con el fin de mostrar una imagen pública responsable (Phyper \& MacLean, 2009).

En su trabajo, Suchman (1995) sugiere que la legitimidad se basa en la coherencia entre los valores declarados por la organización, sus prácticas y normas de comportamiento. En otras palabras, que una compañía busca ajustarse apropiadamente a la sociedad en aras de buscar una especie de licencia social (permiso moral) para poder operar. Por lo tanto, según esta definición, el greenwashing implica una suerte de ausencia de legitimidad no aceptada (Milne \& Patten, 2002). Así las cosas, el greenwashing podría también ser visto como una estrategia de legitimación que se produce cuando las organizaciones de forma voluntaria promueven una impresión de valores sociales y medioambientales incluso cuando no tienen un desempeño tan fuerte en estos términos (Mahoney, Thorne, Cecil, \& LaGore, 2013).

Puntualmente, greenwashing es definido como la brecha entre las acciones sociales corporativas (ASC) sustanciales y simbólicas (Walker \& Wan, 2012). Las ASC simbólicas hacen referencia a lo que la compañía hace con el fin de demostrar a la sociedad comportamientos socialmente responsables; por su parte, las ASC sustanciales se relacionan con las intenciones reales de ejecutar comportamientos socialmente responsables. Un desajuste entre ellas (i.e. la intención de las ASC simbólicas prevalece sobre la intención de las ASC sustanciales) significa la aparición de greenwashing. La forma más común de evidenciar dicha aparición se obtiene al comparar la retórica o los símbolos, con la acción o la sustancia (Lyon \& Montgomery, 2015). Y aunque la mayoría de las veces este fenómeno es percibido como una forma de engaño calculado por parte de las organizaciones, la evidencia también muestra algunos casos en lo que, acercándose a los preceptos de la disonancia cognitiva (Festinger, 1962), una retórica aspiracional podría ser el paso inicial -voluntario o involuntario- para la puesta en práctica posterior de acciones genuinas de RSE (Christensen, Morsing, \& Thyssen, 2013). 
Responsabilidad social empresarial simbólica (RSE Simbólica) y sustancial (RSE Sustancial

En una investigación reciente que evolucionó favorablemente a partir de una ponencia en la Academy of Management Conference de 2015, los trabajos de Donia \& Tretault-Sirsly (2016) y de Donia, Tetrault Sirsly, \& Ronen (2017) lograron desarrollar una propuesta concreta de corte teórico y operacionalización de constructos, en donde se sugiere su vinculación al desarrollo de las actitudes organizacionales en función de la autenticidad de la RSE. Su lógica se basó en la tensión existente entre dos caras de una misma moneda: la RSE Simbólica y la RSE Sustancial.

Evidentemente, sus contribuciones fueron derivadas de los conceptos previamente definidos por Walker \& Wang (2012), con respecto a las ASC simbólicas y sustanciales. Así las cosas, se arguye que mientras que la RSE Simbólica involucra aquellas prácticas que representan cualquier acción relacionada con asuntos de la RSE que una compañía desarrolla para mostrar una especie de formalismo ceremonial, la RSE Sustancial conlleva cambios reales al nivel operacional, que por lo general implican sacrificios tangibles y medibles, que a su vez requieren de una movilización importante de recursos de la organización (Weaver, Trevino, \& Cochran, 1999; Zott \& Huy, 2007). Por lo tanto, el enfoque simplificado de este constructo bi-dimensional tiene sus bases en la diferencia entre la autenticidad o satisfacción de las verdaderas necesidades de la sociedad a partir de la acción de la organización en cuestión (RSE Sustancial), y la falacia o la importancia que se le confiere a la apariencia de suplir dichas necesidades y por lo tanto encuentra beneficios en una mejor reputación ante la sociedad (RSE Simbólica).

\section{Identificación organizacional (OID)}

Con profundas raíces conceptuales ancladas en terrenos del comportamiento organizacional, la OID es originalmente tratada como tópico, definida y desarrollada por Mael \& Ashforth (1992) en un ejercicio académico llevado a cabo con egresados de una institución educativa. Allí, esta actitud fue interpretada como una forma de identificación social, en donde la identidad del individuo es derivada de su clasificación dentro de categorías o grupos sociales. De esta forma, la OID fue originalmente definida como "la percepción de sí mismo en relación con su propio sentido de pertenecer a una organización" (Mael \& Ashforth, 1992; p. 105). En otras palabras, la OID puede ser aquello que define a un individuo con respecto a la organización 
de la cual hace parte. Este constructo es considerado importante en la literatura debido a que -entre otros argumentos de corte más humanístico- ha sido catalogado como un activador positivo de otras actitudes organizacionales (Lee et al., 2015), tales como el compromiso en el trabajo (Riketta, 2005), la satisfacción en el cargo y en el trabajo (Van Dick et al., 2004) y la baja intención de rotación laboral (Cole \& Bruch, 2006).

Se debe reconocer que fue precisamente a partir de los aportes de Mael \& Ashforth que la OID se convirtió en un referente tanto académico como práctico en los estudios organizacionales. Adicionalmente, empezó a ser tratada como una actitud laboral relacionada con el compromiso afectivo de los empleados y su potencial promotor de comportamientos tangibles y colectivos en las organizaciones. De hecho, la importancia académica del concepto ha sido resaltada ampliamente en sendos espacios investigativos. Desde el foro especial que, sobre ella, en conjunto con el concepto de identidad organizacional, se hiciera en el Volumen 25 del American Management Review introducido por Albert, Ashford \& Dutton (2000) hasta las recientes contribuciones de Miscenko \& Day (2016) en donde se hace un interesante esfuerzo de compilación histórica y literaria de este y otros conceptos relacionados.

\section{Asociación entre RSE y OID}

Uno de los evidentes elementos comunes entre los estudios que se han publicado a la fecha, ha sido la determinación del rol de la OID como efecto o como mediador debido a las diferentes formas de presentación del tópico de la RSE (Lee et al., 2015; Riketta, 2005). En este sentido, vale la pena resaltar el impacto generado por autores como Carmeli, Gilat \& Waldman (2007); Grant, Dutton \& Rosso (2008); y Kim, Lee, Lee \& Kim (2010), quienes prepararon el terreno para las futuras contribuciones que han extendido todavía más el conocimiento respectivo. En términos generales, todos los aportes han sido coincidentes con validar una asociación positiva entre estos dos grandes conceptos. Sin embargo, aunque la OID se ha regido básicamente por sus lineamientos originales (Mael \& Ashforth, 1992), el tratamiento que se le ha dado a la RSE si ha gozado de gran diversidad conceptual. Algunos ejemplos de lo anterior pueden ser: percepciones de RSE (Brammer, Millington, \& Rayton, 2007), percepción de la RSE interna vs. externa (De Roeck \& Maon, 2016; Glavas \& Godwin, 2013), actividades o prácticas de RSE (Farooq, Merunka, \& Valette-Florence, 2013; Grant et al., 2008; Peloza \& Papania, 2008), asociaciones de RSE y participación en RSE (Kim et al., 2010; Mozes, Josman, \& Yaniv, 2011), responsabilidad corporativa sobre stakeholders y percepción de apoyo organizacional sobre el medio ambiente (Lamm et al., 2015; Shen, Dumont, Deng, \& 
Deng, 2016), entre otros. La versatilidad de la RSE, por lo tanto, permite la exploración de conceptos más evolucionados y acordes con la problemática real del campo organizacional. Y en ese orden de ideas, tal como se ha mencionado previamente, el presente trabajo rescata la relevancia de explorar la tensión entre las facetas autentica y falaz de la RSE en función de su impacto sobre la OID.

\section{Metodología}

Algunas menciones se pueden encontrar sobre el involucramiento de la autenticidad de la RSE en su relación especifica con actitudes individuales de los empleados y que reclaman por un mayor desarrollo investigativo al respecto (Brammer, He, \& Mellahi, 2015; Glavas \& Godwin, 2013; Rupp, Skarlicki, \& Shao, 2013). Asimismo, se afirma que son necesarios estudios que ayuden a examinar la dinámica de las percepciones de los stakeholders con relación al carácter auténtico e inauténtico de la RSE. Sin embargo, es necesario reconocer que la obra de Donia et al. (2017) ha sido la única que, a la fecha ha hecho una propuesta concreta al respecto. De hecho, en sus manuscritos invitan explícitamente a continuar la línea de estudio particular a partir de la puesta a prueba empírica de sus proposiciones, así como la posibilidad de su estudio en nuevos contextos.

Puntualmente, a la luz del impacto de los conceptos señalados anteriormente sobre las actitudes organizacionales, las proposiciones teóricas de estas autoras son básicamente dos: i) Las atribuciones de la RSE sustancial serán positivamente relacionadas con favorables actitudes por parte de los empleados; y ii) Las atribuciones de la RSE simbólica no tendrán relación o esta relación será negativa con favorables actitudes por parte de los empleados (Donia et al., 2017; Donia \& Tretault-Sirsly, 2016). En ese orden de ideas, y teniendo en cuenta el carácter predictivo del modelamiento a través de ecuaciones estructurales que se desarrolla en el presente estudio, se podría afirmar que, teniendo en cuenta el sexo, la edad y la profesión como variables de control, las primeras hipótesis que se ajustan a la problemática aquí planteada serían básicamente:

H1: Las atribuciones de la RSE sustancial están asociadas positivamente sobre el nivel de OID de los empleados.

H2: Las atribuciones de la RSE simbólica no están asociadas o su asociación es negativa sobre el nivel de OID de los empleados.

Asimismo, se toma en consideración la interacción entre las variables independientes del modelo y el -podríamos decir, lógico- resultado de interdependencia nula o negativa entre 
ellas arrojado en Donia et al. (2017). De esta forma, se buscaría validar que efectivamente los conceptos RSE simbólico y RSE sustancial son contrarios, o al menos independientes entre sí, queriendo decir que las prácticas auténticas de RSE son incompatibles con las prácticas de greenwashing. Consecuentemente, se pone también a prueba la siguiente hipótesis:

H3: La correlación entre la RSE simbólica y la RSE sustancial es negativa o inexistente.

Partiendo de las hipótesis anteriores, para la realización de la presente investigación, se desarrolló un estudio transversal mediante la aplicación de un cuestionario físico auto-administrado, que fue aplicado en el mes de septiembre del 2016. Los datos levantados fueron posteriormente analizados con la ayuda de un modelamiento de ecuaciones estructurales (Wright, 1921) llevado a cabo en lenguaje R versión 3.2.2 (el cual se caracteriza por ser gratuito, libre y de código abierto), lo cual permite evaluar conjuntamente la relación entre una o más variables independientes.

El Modelo de Ecuaciones Estructurales (Structural Equation Model, SEM) es una metodología estadística que parte de proponer la interacción teórica entre variables a partir de un diagrama de rutas (path diagram), determinando finalmente la existencia (o no) de dicha relación a través de su robustez y eventual nivel de significancia. Específicamente, el SEM utiliza un enfoque de análisis factorial confirmatorio o de regresión lineal múltiple, que implica determinar las relaciones lineales entre variables latentes (e.g. constructos teóricos) y observables (e.g. cada componente o ítem de un constructo teórico), en conjunto con relaciones de dependencia o de covarianza entre ellas mismas (Byrne, 1998). El método parte del desarrollo de un modelo de medida con el fin de identificar las variables observables que inciden en las latentes; de tal manera que -y luego de una depuración- se pueda proceder con la determinación de su interacción y la subsecuente obtención de resultados (Manzanares \& Pérez, 2015). Vale la pena resaltar que las principales razones para justificar la selección de la metodología utilizada recaen sobre la capacidad que tiene el SEM de soportar una eventual no normalidad en los datos, así como en la forma en que las cargas del modelo de medida son estimadas mediante un análisis de componentes principales, en donde (garantizando siempre el mismo valor de carga) podrían ser analizadas tantas variables latentes como observadas sean necesarias (Kline, 1994).

\section{Muestra y procedimiento}

El instrumento respectivo fue originalmente aplicado sobre doscientos sesenta y seis profesionales activos laboralmente en Colombia, particularmente en las ciudades de Bogotá, Bucaramanga, Cúcuta y Barrancabermeja. La recolección de datos se hizo efectiva de ma- 
nera física aprovechando el hecho que dichos profesionales eran también participantes de diferentes programas de posgrado de áreas gerenciales de la Escuela de Estudios Industriales y Empresariales de la Universidad Industrial de Santander. Una vez las encuestas fueron diligenciadas y recogidas se realizó una depuración primaria de datos, en la cual se eliminaron cuarenta y tres instrumentos debido a que estaban incompletos o pertenecían a individuos que no tenían vinculación laboral directa con alguna empresa (i.e. muchos de ellos son profesionales independientes y/o contratistas), y se realizó la respectiva foliación de las demás. Posteriormente se tabularon los datos y a partir de estos se realizó una segunda depuración en la cual se eliminaron tres encuestas que presentaban información inconsistente como más de una respuesta en la misma pregunta o un número alto de respuestas en blanco.

Se consolidó finalmente una muestra homogénea de doscientos veinte profesionales vinculados laboralmente a diferentes tipos de empresas con diferentes grados de responsabilidad en sus respectivos trabajos. Todos ellos en su etapa de preparación para el ejercicio gerencial. Por lo tanto, teniendo en cuenta su propia perspectiva académica o experiencial se asume la posesión de, al menos, unos conocimientos básicos en temas relacionados con la dinámica organizacional tales como la RSE y la gestión de talento humano. La muestra estudiada se puede describir como un conjunto de adultos jóvenes con un marcado perfil ingenieril, donde la mayoría son menores de 32 años (64.5\%) y se distribuyen de manera similar entre géneros . En la Figura 1 se consignan las principales características de la muestra a partir del género, edad y profesión; variables de interés para la formación de perfiles socio demográficos

\begin{tabular}{|c|c|c|c|c|c|}
\hline \multicolumn{2}{|c|}{ Género } & \multicolumn{2}{|c|}{ Rango de edad } & \multicolumn{2}{|l|}{ Profesión } \\
\hline & & $22-25$ & $20,3 \%$ & Ingeniero Industrial & $27,7 \%$ \\
\hline & & $25-28$ & $29,5 \%$ & Ingeniero Civil & $9,5 \%$ \\
\hline \multirow[t]{3}{*}{ Femenino } & $47,0 \%$ & $28-31$ & $14,7 \%$ & Administrador de Empresas & $7,3 \%$ \\
\hline & & $31-34$ & $12,0 \%$ & Ingeniero Mecánico & $5,5 \%$ \\
\hline & & $34-37$ & $7,8 \%$ & Ingeniero Electricista & $4,5 \%$ \\
\hline \multirow[t]{3}{*}{ Maculino } & $52,5 \%$ & $37-40$ & $6,5 \%$ & Ingeniero Químico & $3,6 \%$ \\
\hline & & $40-43$ & $1,8 \%$ & Ingeniero de Sistemas & $3,2 \%$ \\
\hline & & $43-46$ & $2,8 \%$ & Contador Público & $3,2 \%$ \\
\hline \multirow[t]{5}{*}{$\mathrm{N} / \mathrm{R}$} & $0,5 \%$ & $46-49$ & $2,3 \%$ & Ingeniero de petroleos & $2,7 \%$ \\
\hline & & $49-52$ & $0,5 \%$ & Ingeniero & $2,3 \%$ \\
\hline & & $52-55$ & $0,9 \%$ & Ingeniero Electromecánico & $2,3 \%$ \\
\hline & & $55-59$ & $0,9 \%$ & Ingeniero Financiero & $2,3 \%$ \\
\hline & $100,0 \%$ & & $100,0 \%$ & & $74,1 \%$ \\
\hline
\end{tabular}

Figura 1. Distribución de las principales variables de control Fuente: Elaboración propia. 


\section{Medidas e instrumento}

Adicional a las variables de control definidas previamente: sexo, edad y principales profesiones (las cuales no presentaron diferencia estadística), la encuesta en mención midió tres variables latentes o constructos: RSE sustancial, RSE simbólica y OID, cuyas variables observables o ítems eran calificados por los encuestados a través de una escala Likert de 1 a 5, siendo 1 : completamente en desacuerdo y 5: completamente de acuerdo. Para la medición de la OID, se acudió a lo que se podría denominar como el 'golden standard' en la calibración de este constructo: una escala de seis ítems propuesta por Mael \& Ashforth (1992) altamente citada y validada en la literatura relevante. Asimismo, para las mediciones de RSE simbólica y RSE sustancial se tuvo en cuenta el trabajo de Donia et al. (2017), en donde para la primera se trabajó con una escala de seis ítems, y para la segunda con una escala de ocho ítems.

De esta forma el instrumento respectivo incluyó un total de veinte variables observables estructuradas en forma de frase o enunciado. De la codificación descrita en el Anexo 7.2, el conjunto de enunciados del 2.1.1 a 2.1.8 miden el grado de RSE sustancial, de las preguntas 2.2.1 a 2.2.6 el nivel de RSE simbólica y de las preguntas 3.1 a 3.6, la OID. El orden de dichos ítems fue aleatoriamente alterado para efectos de reducción de la percepción de monotonía en las respuestas por parte de los encuestados. La relación entre las variables se registra en el Anexo 7.1, los enunciados están plasmados en el Anexo 7.2 y un resumen de las variables en la Tabla 1.

Tabla 1

Resumen de las variables del modelo

\begin{tabular}{llllllllll}
\hline Tipo de Variable & Codificación & & & & \\
\hline \multirow{3}{*}{ Observadas (independientes) } & 2.1 .1 & 2.1 .2 & 2.1 .3 & 2.1 .4 & 2.1 .5 & 2.1 .6 & 2.1 .7 & 2.1 .8 & (RSE Sustancial) \\
& 2.1 & 2.2 .2 & 2.2 .3 & 2.2 .4 & 2.2 .5 & 2.2 .6 & & (RSE Simbólica) \\
& 3.1 & 3.2 & 3.3 & 3.4 & 3.5 & 3.6 & & (OID) \\
& Responsabilidad Social Empresarial Sustancial & & \\
Latentes (dependientes) & Responsabilidad Social Simbólica & & \\
& Identificación Organizacional & & \\
& Género & & & & \\
De control (independientes) & Rango de edad & & & & & \\
& & & & & & \\
\end{tabular}

Fuente: Elaboración propia. 
Se debe mencionar que previamente a la aplicación oficial del instrumento, tanto su versión en inglés como en español fueron sometidas a revisión externa por parte de cinco académicos expertos en Francia, España y Colombia. Sus áreas de experticia iban desde temas relacionados con RSE y comportamiento organizacional hasta diseño de cuestionarios para investigación. De esta forma, la versión final aplicada recogió sus observaciones.

Una vez aplicada la encuesta y previo a iniciar un análisis factorial exploratorio (AFE) se procedió a verificar la fiabilidad del instrumento; para lo cual se recurrió al coeficiente Alfa de Cronbach. Así mismo se comprobó que las características de covariabildad de los datos fueran pertinentes para realizar un análisis de reducción de dimensión; para estos propósitos se utilizó la medida de adecuación muestral de Kaiser-Meyer-Olkin (KMO) y la prueba de esfericidad de Bartlett (EB). En la Tabla 2 se comparan los valores del estudio con los parámetros de cumplimiento de las pruebas estadísticas propuestas para verificar la pertinencia de realizar un AFE.

Tabla 2

Estudio de pertinencia de AFE

\begin{tabular}{llllll}
\hline & \multirow{2}{*}{ Alfa de Cronbach } & \multirow{2}{*}{ KMO } & \multicolumn{3}{l}{ Prueba de esfericidad de Bartlett } \\
\cline { 5 - 6 } & & & Aprox. Chi-cuadrado & GL & Significancia \\
\hline Valor recomendado & $>0.8$ & $>0.5$ & - & - & $<0.05$ \\
Valor del Modelo & 0.889 & 0.889 & 2126.566 & 190 & 0.00 \\
\hline
\end{tabular}

Fuente: Elaboración propia.

\section{Resultados}

El AFE se realiza eligiendo tres factores mediante el criterio de Kaiser (1960), por el método de componentes principales sin encontrar diferencia estadística significativa entre las variables de control, logrando explicar así el $60.7 \%$ de la varianza de los datos. Del dataset original no son tenidas en cuenta las preguntas 2.2.3, 2.2.6 y 3.6 (dos preguntas relacionadas con la RSE simbólica y una con la OID) debido a que sus cargas no se ajustaban al modelo de tres factores propuesto ( $s u$ valor no era lo suficientemente alto: $c \geq 0.5$ para influir en el factor asignado), decisión soportada por un análisis factorial confirmatorio o modelo estructural de medida. En la Tabla 3 se observan las relaciones entre variables observadas y los constructos (cargas). Cabe resaltar que, según el valor de las cargas, el factor 2 (OID) presenta valores negativos pero altos (en valor absoluto superior a 0.5 ). 
Tabla 3

AFE con 3 factores

\begin{tabular}{lllllllll}
\hline Variable & 2.1 .6 & 2.1 .1 & 2.1 .3 & 2.1 .8 & 2.1 .2 & 2.1 .7 & 2.1 .4 & 2.1 .5 \\
RSE sustancial (factor1) & 0.82 & 0.82 & 0.81 & 0.78 & 0.71 & 0.71 & 0.70 & 0.50 \\
Variable & 3.5 & 3.1 & 3.2 & 3.4 & 3.3 & & & \\
OID (factor2) & -0.81 & -0.70 & -0.69 & -0.67 & -0.54 & & & \\
Variable & 2.2 .1 & 2.2 .2 & 2.2 .4 & 2.2 .5 & & & & \\
RSE simbólica (factor3) & 0.87 & 0.85 & 0.70 & 0.66 & & & & \\
\hline
\end{tabular}

Fuente: Elaboración propia.

A través de los paquetes estadísticos sem y lavaan del software R, se evaluó la ecuación estructural propuesta, determinando con alta significancia estadística (goodness of fit index de 0.929) que la identificación organizacional puede ser descrita y formada a partir de la percepción sustancial de RSE desarrollada en las empresas (el valor de la pendiente de la RSE Sustancial es de 8.805), además, si bien la RSE auténtica y falaz percibida por los profesionales covaría (el p value de la influencia de la RSE Simbólica es significativo y existe correlación entre las variables del Anexo 7.1), no existe suficiente información estadística para afirmar un significativo grado de relación (ni positiva, ni negativa) entre ésta última respecto a la OID. De la misma forma, se encuentra una correlación directa y con significancia estadística entre la RSE sustancial y la RSE simbólica (valor estimado de la correlación entre los constructos es de 0.178), que contradice H3. Los resultados obtenidos se resumen en la Tabla 4.

Tabla 4

Resultados de las hipótesis evaluadas

\begin{tabular}{lllll}
\hline & & Estimado & $p$ value & Resultado \\
\hline H1 & RSESust $=>$ OID & 0.805 & 0.000 & Hipótesis Soportada \\
H2 & RSESimb $=>$ OID & -0.047 & 0.000 & Hipótesis Soportada \\
H3 & RSESust $<=$ RSESimb & 0.178 & 0.003 & Hipótesis Rechazada \\
\hline
\end{tabular}

Fuente: Elaboración propia.

Asimismo, el modelo representado en la Figura 2 presenta una aproximación gráfica de los hallazgos. 


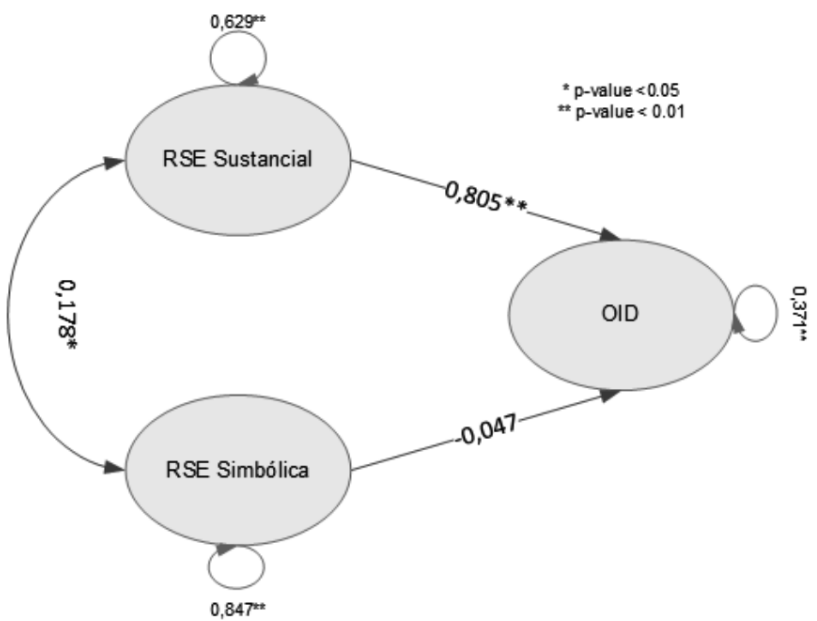

Figura 2. Representación del modelo estructural propuesto

Fuente: Elaboración propia.

En la Tabla 4 se registran los índices o parámetros de ajuste del modelo respectivo, encontrando sólo en la prueba de Chi-cuadrado un valor que cae en la zona de ajuste bajo; sin embargo, este comportamiento puede deberse a que dicha prueba es sensible al tamaño muestral, por tanto, al aumentar este último, aumenta a la par la probabilidad de rechazar la hipótesis de ajuste (Bagozzi \& Yi, 1988). Teniendo en cuenta lo anterior se analizan otros índices de estimación (considerando grados de libertad y complejidad del modelo) que dan muestra de un ajuste adecuado del modelo propuesto. Además, se calcula la raíz media cuadrada del error de aproximación (RMSEA), el cual se puede interpretar como el error de aproximación medio por grado de libertad. Se concluye por tanto un ajuste adecuado del modelo en cuestión. 
Tabla 5

AFE con 3 factores

\begin{tabular}{llll}
\hline Parámetro de ajuste & Recomendado & Valor del modelo & Ajuste del modelo \\
\hline Chi-square & $\mathrm{p} \geq 0.05$ & $144.023(\mathrm{p}=0.006)$ & Bajo \\
Chi-square/degree of freedom & $\leq 5.00$ & $1.380(104 \mathrm{df})$ & Bueno \\
GFI (goodness of fit index) & $\geq 0.90$ & 0.929 & Bueno \\
AGFI (adjusted goodness of fit & & 0.896 & \\
index) & $\geq 0.90$ & & Moderado \\
RMSEA (root mean square error & & & \\
of approximation) & $\leq 0.08$ & 0.042 & Bueno \\
NFI (normed fit index) & $\geq 0.90$ & 0.922 & Bueno \\
NNFI (Non-normed fit index) & $\geq 0.90$ & 0.970 & Bueno \\
CFI (Comparative fit index) & $\geq 0.90$ & 0.977 & Bueno \\
\hline
\end{tabular}

Fuente: Elaboración propia.

\section{Discusión}

Estudios previos se han concentrado en la relación entre la RSE (y sus diferentes manifestaciones) con diferentes actitudes laborales de los empleados, encontrando relaciones positivas entre los constructos analizados. De esta forma, se ha consolidado la idea de un efecto positivo generado por parte de la RSE sobre la OID en el ambiente laboral (Glavas \& Godwin, 2013; Jones \& Rupp, 2017). En otras palabras, la literatura se ha encargado de presentar evidencia suficiente para afirmar que las prácticas de RSE efectivamente mejoran la forma en que un empleado se identifica con su organización. Sin embargo, dichos matices no han tenido en cuenta la búsqueda de legitimación de dichas prácticas organizacionales. El presente estudio se preocupó por esto último, de tal forma que evaluó el carácter auténtico (i.e. sustancial) y falaz (i.e. simbólico) de la RSE y el efecto que ellos tienen sobre la OID de los empleados.

Los resultados obtenidos fueron de naturaleza variada. Por una parte, se observó una dependencia positiva fuerte y significativa entre la RSE sustancial y la OID, así como una desconexión causal entre la RSE simbólica y la OID (soportando las hipótesis 1 y 2). Por otra parte, se encontró una correlación positiva débil entre la RSE sustancial y la RSE simbólica (rechazando la hipótesis 3). El análisis que de ellos se puede hacer se divide en dos aseveraciones fundamentales. La primera es la ratificación inicial de dos de las proposiciones al modelo teórico propuesto por Donia \& Tretault-Sirsly (2016) que posteriormente sirvió como insumo a la validación y operacionalización de constructos (Donia et al., 2017). La 
segunda es una inesperada contradicción de resultados en donde se pudo observar que dos conceptos aparentemente contrarios se correlacionan directamente, máxime cuando en las pruebas iniciales de validación de estas autoras, la correlación encontrada entre ellos era -en apariencia lógicamente- de naturaleza inversa o nula.

En cuanto a lo primero, se reporta coincidencia con lo planteado desde la teoría. Por ello se podría pensar que un empleado efectivamente es capaz de percibir y procesar señales derivadas de las prácticas de RSE emitidas por su propia organización y diferenciarlas entre prácticas auténticas (sustanciales) y falaces (simbólicas), así como apropiarlas de manera independiente para efectos de moldear o no sus actitudes (en este caso, su OID). Con respecto a lo segundo, lo que se pudo observar fue básicamente una especie de consentimiento a la adopción organizacional de prácticas falaces de RSE por parte de los empleados en un contexto de auto-definición como miembros de una organización.

A pesar de haber hecho mención tácita de ellas, las implicaciones tanto teóricas como prácticas del estudio realizado son de naturaleza diversa. En cuanto a lo teórico, se avanzó en el conocimiento que se tiene tanto en el campo de estudio de la micro RSE como en los aspectos conceptuales que rodean la teoría de la legitimidad sobre los empleados, especialmente cuando dicha legitimidad es defendida a través del engaño (característico del greenwashing). Adicionalmente, según el saber y entender de los autores, este trabajo se constituye en la primera validación parcial empírica de un modelo teórico recientemente propuesto. El hecho mismo de haber ejecutado la investigación propuesta por Donia \& Tretault-Sirsly (2016) en un contexto de un país latinoamericano y de haber encontrado resultados llamativos y en parte disimiles a la propuesta teórica original, abre la puerta para identificar matices en la teoría formulada que podrían hacer pensar en el contexto como elemento explicativo de los resultados (Jamali \& Karam, 2016; Rodrigo et al., 2016). Sin embargo, hay que reconocer que esto último requiere un mayor nivel de profundización investigativa para llegar a conclusiones más definitivas.

Desde lo práctico, se observa la posibilidad de capitalizar estos resultados a la luz de las decisiones organizacionales que se relacionen con la RSE junto con su divulgación a los diferentes sectores de la sociedad. La evidencia obtenida nos sugiere que los empleados parecen no ser ajenos a estas señales y son capaces de procesarlas en aras de moldear sus actitudes (percepciones, intenciones y comportamientos) en el ambiente de trabajo. Esto quiere decir que, si el verdadero concepto de RSE logra ser manipulado por parte de los tomadores de decisión, las consecuencias podrían no ser tan convenientes a nivel de su fuerza laboral, y por lo tanto tener repercusiones en términos de la sostenibilidad de la sociedad en general, y de las organizaciones en particular. 
Una de las aparentes limitaciones del presente estudio tiene que ver con el enfoque casi exclusivo del instrumento utilizado sobre las cuestiones sociales de la RSE, lo cual podría dejar de lado los aspectos ambientales de la misma. Lo anterior, a pesar de suponer una salvedad a las contribuciones obtenidas, es justificado previamente por Donia et al. (2016), ya que ellas plantean explícitamente la equivalencia de la contraprestación social con la protección al medio ambiente por parte de las organizaciones, a la luz de las percepciones de sus empleados. Sin embargo, un ejercicio que podría proyectar frentes de investigación más específicos, sería la delimitación clara de las dimensiones sociales, ambientales e incluso económicas de la RSE. Asimismo, tal como lo hicieron previamente Amato, Busraschi \& Peretti (2016), el hecho de abordar de manera diferenciada las nociones de RSE y el de sostenibilidad (o sustentabilidad como también es conocida), es algo que podría agregarle más valor a lo discutido en el presente estudio, toda vez que este último concepto es algunas veces percibido como de mayor trascendencia que la RSE misma.

Igualmente, otro punto de discusión se encuentra representado en la naturaleza transversal de la medición respectiva, lo cual no permitiría afirmar categóricamente las relaciones de causalidad sugeridas. Sin embargo, ésta se intentó atenuar con la utilización del modelamiento de ecuaciones estructurales así como en un fuerte soporte teórico que en parte permite predecir dichas relaciones en el modelo conceptual planteado. De la misma forma, también se reconoce la imposibilidad de garantizar la generalización (o validez externa) de los resultados obtenidos, teniendo en cuenta las características de la muestra trabajada. No obstante, el equilibrio generado por la utilización de muestras similares en estudios previos (Brammer et al, 2015; Carmeli et al, 2007; Kim et al., 2010) junto con la homogeneidad en el perfil de los integrantes de la muestra aquí examinada, son elementos que brindan cierta tranquilidad en aras de reducir potenciales sesgos en el estudio.

A pesar de lo comentado previamente, también se puede despertar cierta controversia con respecto al contexto en donde fue desplegado. En este sentido, es posible que haber recopilado los datos en Colombia, haga que los resultados sean relevantes de diferentes maneras en otros contextos culturales. Sin embargo, de ser ese el caso, además de contribuir a una mejor comprensión del proceso especifico en dicho país, este estudio también abre la puerta al contraste y comparación futura de sus resultados con otros contextos -similares o no- con el fin de producir un conocimiento más preciso y amplio sobre el tema. Tanto la RSE como la naturaleza de los individuos que hacen parte de las organizaciones son conceptos con matices territoriales y su análisis no puede pasar por alto aquellas variables que las separen del resto. Por ejemplo, Colombia, al igual que muchos otros estados latinoamericanos, tiene una condición de país biodiverso y en camino de su desarrollo; sin embargo, presenta profundos 
problemas sociales que hacen que los resultados de este tipo de estudios sean realmente un reto en términos de su interpretación y puesta en práctica. En este sentido, el engaño corporativo sobre las prácticas de RSE es algo que no debería tolerarse en este tipo de regiones, ya que las implicaciones de hacerlo pueden ser costosas para su bienestar presente y futuro.

Adicional al afinamiento de las limitaciones anteriores, futuras investigaciones se podrían concentrar en la validación de las relaciones entre la RSE sustancial y la RSE simbólica con otros constructos actitudinales propios del campo del comportamiento organizacional al nivel del individuo, incluyendo, si así se considera conveniente, a la OID a través de su carácter mediador. Asimismo, la posibilidad de involucrar variables moderadoras y/o más variables mediadoras es una oportunidad que siempre estará presente para lograr avanzar más en el conocimiento recabado. También se abre la puerta para que a través de proyectos de investigación cualitativa se puedan encontrar pistas más profundas que agreguen a la definición del greenwashing en un contexto similar a los típicamente estudiados en la literatura. En este sentido, casos de estudio, análisis de contenidos o trabajos etnográficos en países emergentes podrían representar ejemplos excepcionales que aporten aún más al entendimiento integral de los comportamientos de los empleados en entornos organizacionales.

\section{Conclusiones}

La evidencia recabada en el presente estudio invita a pensar que los empleados pueden percibir positivamente las prácticas sustanciales de RSE y ser indiferentes ante aquellas prácticas de RSE simbólicas. Lo anterior se refleja al evaluar su efecto sobre la identificación que ellos mismos perciben como miembros de sus organizaciones. Sin embargo, teniendo en cuenta estudios previos, se podría inferir también que otra serie de resultados actitudinales como su satisfacción laboral, su compromiso e incluso su efectividad podrían depender de dichas percepciones.

Por otra parte, un hallazgo inquietante que desafía los supuestos iniciales de las proposiciones teóricas utilizadas (Donia \& Tretault-Sirsly, 2016), es que de alguna manera estos empleados también vindican la utilización de símbolos, ceremonias y formalismos para legitimar las prácticas (muchas veces auténticas) de RSE de sus organizaciones. En otras palabras, se percibe una especie de validación del discurso aspiracional positivo (Christensen et al., 2013) en donde en principio parecería que no habría mucha polémica. Se debe aceptar que las organizaciones están en su derecho de hacer uso de diversos mecanismos para divulgar el cumplimiento de un propósito orientado a la RSE cuando ésta ejerce presencia genuina en los aspectos organizacionales (políticas, procesos, operaciones y metas, entre otros), además 
de hacer uso de los mismos para reforzar sus posturas y definir objetivos (Berrone, 2016).

No obstante, lo encontrado pudiera ser también una clara señal de preocupación en donde el concepto de RSE hubiese podido ser alterado de acuerdo a experiencias pasadas de tipo académico o laboral. Inquieta por tanto que se reconozcan como RSE, algunas prácticas vacías como la validación del discurso sin acción, las certificaciones ambientales buscando solo el cumplimiento de un requisito, las donaciones buscando beneficios tributarios o incluso el hecho de alegar aportes al desarrollo de la sociedad a través del cumplimiento de la legalidad. Todas ellas representan formas de greenwashing a través del uso de mecanismos sutiles que tratan de crear una imagen alterada de la realidad (Mahoney et al., 2013; Milne \& Patten, 2002). El hecho es que esta eventual situación implicaría un desafío enorme a nivel educacional para redirigir esfuerzos en aras de dejar clara la definición real de RSE (Berrone, 2016). Y aunque esto último es un tema transversal de todos los niveles y para todas las áreas del conocimiento, es especialmente delicado a nivel de formación gerencial, toda vez que son los participantes aquellos encargados de tomar las decisiones en las organizaciones destinadas a dirigir en el futuro.

\section{Referencias}

Aguilera, R. V., Rupp, D. E., Williams, C. A., \& Ganapathi, J. (2007). Putting the s back in corporate social responsibility: A multilevel theory of social change in organizations. Academy of Management Review, 32(3), 836-863. https://doi.org/10.5465/AMR.2007.25275678

Aguinis, H., \& Glavas, A. (2012). What we know and don't know about Corporate Social Responsibility. Journal of Management, 38(4), 932-968. https://doi.org/10.1177/0149206311436079

Albert, S., Ashforth, B. E., \& Dutton, J. E. (2000). Organizational identity and identification: Charting new waters and building new bridges. Academy of Management Review, 25(1), 13-17. https://doi.org/10.5465/ AMR.2000.2791600

Amato, C. N., Buraschi, M., \& Peretti, M. F. (2016). Orientación de los empresarios de Córdoba-Argentina hacia la sustentabilidad y la responsabilidad social empresarial: identificación de variables asociadas a cada constructo. Contaduría y Administración, 61, 84-105. http://dx.doi.org/10.1016/j.cya.2015.10.001

Bagozzi, R. P., \& Yi, Y. (1988). On the evaluation of structural equation models. Journal of the Academy of Marketing Science, 16(1), 74-94. https://doi.org/10.1007/BF02723327

Berrone, P. (2016). Green Lies: How Greenwashing can destroy a company (and how to go green without the wash). IESE Insight.

Boudreau, J. W., \& Ramstad, P. M. (2005). Talentship talent segmentation, and sustainability: A new hr decision science paradigm for a new strategy definition. Human Resource Management, 44(2), 129-136. https://doi. org/10.1002/hrm.20054

Brammer, S., He, H., \& Mellahi, K. (2015). Corporate Social Responsibility, Employee Organizational Identification, and Creative Effort. Group \& Organization Management, 40(3), 323-352. https://doi. org/10.1177/1059601114562246

Brammer, S. J., Millington, A., \& Rayton, B. (2007). The contribution of corporate social responsibility to organizational commitment, 18(10), 1701-1719. https://doi.org/10.1080/09585190701570866 


\section{O. E. Contreras-Pacheco, et al./ Contaduría y Administración 64(4), 2019, 1-24}

http://dx.doi.org/10.22201/fca.24488410e.2018.1631

Byrne, B. M. (1998). Structural equation modeling with LISREL, PRELIS, and SIMPLIS : basic concepts, applications, and programming. Multivariate Applications Book Series. https://doi.org/10.4324/9780203774762

Carmeli, A., Gilat, G., \& Waldman, D. A. (2007). The role of perceived organizational performance in organizational identification, adjustment and job performance. Journal of Management Studies, 44, 972-992. https:// doi.org/10.1111/j.1467-6486.2007.00691.x

Christensen, L. T., Morsing, M., \& Thyssen, O. (2013). CSR as aspirational talk. Organization, 20(3), 372-393. https://doi.org/10.1177/1350508413478310

Cole, M. S., \& Bruch, H. (2006). Organizational identity strength, identification, and commitment and their relationships to turnover intention: does organizational hierarchy matter? Journal of Organizational Behavior, 27(5), 585-605. https://doi.org/10.1002/job.378

De Roeck, K., \& Maon, F. (2016). Building the Theoretical Puzzle of Employees' Reactions to Corporate Social Responsibility: An Integrative Conceptual Framework and Research Agenda. Journal of Business Ethics, 1-17. https://doi.org/10.1007/s10551-016-3081-2

Delmas, M. A., \& Pekovic, S. (2013). Environmental standards and labor productivity: Understanding the mechanisms that sustain sustainability. Journal of Organizational Behavior, 34(2), 230-252. https://doi.org/10.1002/job.1827

Donia, M. B. L., Tetrault Sirsly, C.-A., \& Ronen, S. (2017). Employee Attributions of Corporate Social Responsibility as Substantive or Symbolic: Validation of a Measure. Applied Psychology, 66(1), 103-142. https://doi. org/10.1111/apps.12081

Donia, M. B. L., \& Tretault-Sirsly, C.-A. (2016). Determinants and consequences of employee attributions of corporate social responsibility as substantive or symbolic. European Management Journal, 34(3), 232-242. https://doi.org/10.1016/j.emj.2016.02.004

Elkington, J. (1998). Partnerships from cannibals with forks: The triple bottom line of 21 st-century business. Environmental Quality Management, 8(1), 37-51. https://doi.org/10.1002/tqem.3310080106

Evans, W. R., \& Davis, W. (2014). Corporate Citizenship and the Employee: An Organizational Identification Perspective. Human Performance, 27(2), 129-146. https://doi.org/10.1080/08959285.2014.882926

Evans, W. R., Davis, W., \& Frink, D. D. (2011). An Examination of Employee Reactions to Perceived Corporate Citizenship1. Journal of Applied Social Psychology, 41(4), 938-964. https://doi.org/10.1111/j.15591816.2011.00742.x

Farooq, O., Merunka, D., \& Valette-Florence, P. (2013). Employees' Response to Corporate Social Responsibility: An Application of a Non Linear Mixture REBUS Approach. In Springer New York (pp. 257-268). https://doi. org/10.1007/978-1-4614-8283-3_18

Festinger, L. (1962). A theory of cognitive dissonance (2nd ed.). Stanford: Stanford university press.

Freeman, R. E. E., \& McVea, J. (2006). The Blackwell Handbook of Strategic Management. In M. A. Hitt, R. E. Freeman, \& J. S. Harrison (Eds.), The Blackwell Handbook of Strategic Management (pp. 189-207). Oxford, UK: Blackwell Publishing Ltd. https://doi.org/10.1111/b.9780631218616.2006.00010.x

Glavas, A., \& Godwin, L. N. (2013). Is the Perception of "Goodness" Good Enough? Exploring the Relationship Between Perceived Corporate Social Responsibility and Employee Organizational Identification. Journal of Business Ethics, 114(1), 15-27. https://doi.org/10.1007/s10551-012-1323-5

Godfrey, P. C. (2005). The relationship between corporate philanthropy and shareholder wealth: A risk management perspective. Academy of Management Review, 30(4), 777-798. Retrieved from http://www.jstor.org/ stable/20159168

Grant, A. M., Dutton, J. E., \& Rosso, B. D. (2008). Giving Commitment: Employee Support Programs and The Prosocial Sensemaking Process. Academy of Management Journal, 51(5), 898-918. https://doi.org/10.5465/ AMJ.2008.34789652

Jamali, D., \& Karam, C. (2016). Corporate Social Responsibility in Developing Countries as an Emerging Field of Study. International Journal of Management Reviews, 1-30. https://doi.org/10.1111/ijmr.12112

Jones, C., \& Volpe, E. H. (2011). Organizational identification: Extending our understanding of social identities through social networks. Journal of Organizational Behavior, 32(3), 413-434. https://doi.org/10.1002/job.694 
Jones, D. A., \& Rupp, D. E. (2017). Social responsibility IN and OF organizations: the psychology of corporate social responsibility among organizational members. In D. S. Ones, N. Anderson, C. Viswesvaran, \& H. K. Sinangil (Eds.), The SAGE Handbook of Industrial, Work, and Organizational Psychology V3. Thousand Oaks: SAGE Publications, Inc. https://doi.org/10.4135/9781473914964.n16

Kaiser, H. F. (1960). The Application of Electronic Computers to Factor Analysis. Educational and Psychological Measurement, 20(1), 141-151. https://doi.org/10.1177/001316446002000116

Kim, H.-R., Lee, M., Lee, H.-T., \& Kim, N.-M. (2010). Corporate social responsibility and employee-company identification. Journal of Business Ethics, 95(4), 557-569. https://doi.org/10.1007/s10551-010-0440-2

Kline, P. (1994). An easy guide to factor analysis. Personality and Individual Differences, 17(2), 302. https://doi. org/10.1016/0191-8869(94)90040-X

Koehn, D. (1995). A Role for Virtue Ethics in the Analysis of Business Practice. Business Ethics Quarterly, 5(3), 533-539. https://doi.org/10.2307/3857397

Lamm, E., Tosti-Kharas, J., \& King, C. E. (2015). Empowering Employee Sustainability: Perceived Organizational Support Toward the Environment. Journal of Business Ethics, 128(1), 207-220. https://doi.org/10.1007/ s10551-014-2093-z

Lee, E.-S., Park, T.-Y., \& Koo, B. (2015). Identifying organizational identification as a basis for attitudes and behaviors: A meta-analytic review. Psychological Bulletin, 141(5), 1049-1080. https://doi.org/10.1037/bul0000012

Lyon, T. P., \& Montgomery, A. W. (2015). The Means and End of Greenwash. Organization \& Environment, 28(2), 223-249. https://doi.org/10.1177/1086026615575332

Mael, F., \& Ashforth, B. E. (1992). Alumni and their alma mater: A partial test of the reformulated model of organizational identification. Journal of Organizational Behavior, 13(2), 103-123. https://doi.org/10.1002/ job. 4030130202

Mahoney, L. S., Thorne, L., Cecil, L., \& LaGore, W. (2013). A research note on standalone corporate social responsibility reports: Signaling or greenwashing? Critical Perspectives on Accounting, 24(4-5), 350-359. https:// doi.org/10.1016/j.cpa.2012.09.008

Manzanares, F. V., \& Pérez, J. E. S. (2015). El impacto de los intangibles estratégicos en el desempeño exportador de la PYME manufacturera. Intangible Capital, 11(1), 13-40. https://doi.org/10.3926/ic.567

Meyer, J. W., \& Rowan, B. (1977). Institutionalized Organizations: Formal Structure as Myth and Ceremony. American Journal of Sociology, 83(2), 340-363. https://doi.org/10.1086/226550

Milne, M. J., \& Patten, D. M. (2002). Securing organizational legitimacy. Accounting, Auditing \& Accountability Journal, 15(3), 372-405. https://doi.org/10.1108/09513570210435889

Miscenko, D., \& Day, D. V. (2016). Identity and identification at work. Organizational Psychology Review, 6(3), 215-247. https://doi.org/10.1177/2041386615584009

Mozes, M., Josman, Z., \& Yaniv, E. (2011). Corporate social responsibility organizational identification and motivation. Social Responsibility Journal, 7(2), 310-325. https://doi.org/10.1108/17471111111141558

Peloza, J., \& Papania, L. (2008). The Missing Link between Corporate Social Responsibility and Financial Performance: Stakeholder Salience and Identification. Corporate Reputation Review, 11(2), 169-181. https://doi. org/10.1057/crr.2008.13

Phyper, J. D., \& MacLean, P. (2009). Good to green: managing business risks and opportunities in the age of environmental awareness. John Wiley \& Sons.

Ramus, C. A., \& Montiel, I. (2005). When Are Corporate Environmental Policies a Form of Greenwashing? Business \& Society, 44(4), 377-414. https://doi.org/10.1177/0007650305278120

Riketta, M. (2005). Organizational identification: A meta-analysis. Journal of Vocational Behavior, 66(2), 358-384. https://doi.org/10.1016/j.jvb.2004.05.005

Rodrigo, P., Duran, I. J., \& Arenas, D. (2016). Does it really pay to be good, everywhere? A first step to understand the corporate social and financial performance link in Latin American controversial industries. Busines Ethics: A European Review, 25(3), 286-309. https://doi.org/10.1111/beer.12119 
Rupp, D. E., \& Mallory, D. B. (2015). Corporate Social Responsibility: Psychological, Person-Centric, and Progressing. Annual Review of Organizational Psychology and Organizational Behavior, 2, 211-236. https://doi. org/10.1146/annurev-orgpsych-032414-111505

Rupp, D. E., Skarlicki, D., \& Shao, R. (2013). The Psychology of Corporate Social Responsibility and Humanitarian Work: A Person-Centric Perspective. Industrial and Organizational Psychology, 6(4), 361-368. https://doi. org/10.1111/iops.12068

Shen, J., Dumont, J., Deng, X., \& Deng, X. (2016). Employees Perceptions of Green HRM and Non-Green Employee Work Outcomes: The Social Identity and Stakeholder Perspectives. Group \& Organization Management, 1059601116664610. https://doi.org/10.1177/1059601116664610

Suchman, M. C. (1995). Managing Legitimacy: Strategic and Institutional Approaches. Academy of Management Review, 20(3), 571-610. Retrieved from http://www.jstor.org/stable/258788

United Nations World Commission on Environment and Development. (1987). The Brundtland report: "Our common future." Medicine and War, 4(1), 17-25. https://doi.org/10.1080/07488008808408783

Van Dick, R., Christ, O., Stellmacher, J., Wagner, U., Ahlswede, O., Grubba, C., \& Tissington, P. A. (2004). Should I stay or should I go? Explaining turnover intentions with organizational identification and job satisfaction. British Journal of Management, 15(4), 351-360. https://doi.org/10.1111/j.1467-8551.2004.00424.x

Walker, K., \& Wan, F. (2012). The Harm of Symbolic Actions and Green-Washing: Corporate Actions and Communications on Environmental Performance and Their Financial Implications. Journal of Business Ethics, 109, 227-242. https://doi.org/10.1007/s10551-011-1122-4

Weaver, G. R., Trevino, L. K., \& Cochran, P. L. (1999). Integrated and decoupled corporate social performance: Management commitments, external pressures, and corporate ethics practices. Academy of Management Journal, 42(5), 539-552. https://doi.org/10.2307/256975

Wright, S. (1921). Correlation and causation. Journal of Agricultural Research, 20(7), 557-585.

Zott, C., \& Huy, Q. N. (2007). How Entrepreneurs Use Symbolic Management to Acquire Resources. Administrative Science Quarterly, 52(1), 70-105. https://doi.org/10.2189/asqu.52.1.70

\section{Anexos}

\section{Tabla A1}

Matriz de correlación entre variables observadas para el modelo estructural

\begin{tabular}{|c|c|c|c|c|c|c|c|c|c|c|c|c|c|c|c|c|c|}
\hline & $P 2.1 .1$ & $P 2.1 .2$ & $P 2.1 .3$ & $P 2.1 .4$ & $P 2.1 .5$ & P2.1.6 & $P 2.1 .7$ & $P 2.1 .8$ & $P 2.2 .1$ & $P 2.2 .2$ & $P 2.2 .4$ & $P 2.2 .5$ & P3.1 & P3.2 & P3.3 & P3.4 & $P 3.5$ \\
\hline P2.1.1 & 1,00 & & & & & & & & & & & & & & & & \\
\hline$P 2.1 .2$ & 0,62 & 1,00 & & & & & & & & & & & & & & & \\
\hline$P 2.1 .3$ & 0,75 & 0,56 & 1,00 & & & & & & & & & & & & & & \\
\hline$P 2.1 .4$ & 0,44 & 0,50 & 0,45 & 1,00 & & & & & & & & & & & & & \\
\hline$P 2.1 .5$ & 0,42 & 0,39 & 0,42 & 0,35 & 1,00 & & & & & & & & & & & & \\
\hline$P 2.1 .6$ & 0,79 & 0,60 & 0,73 & 0,48 & 0,42 & 1,00 & & & & & & & & & & & \\
\hline P2.1.7 & 0,63 & 0,55 & 0,71 & 0,41 & 0,37 & 0,59 & 1,00 & & & & & & & & & & \\
\hline$P 2.1 .8$ & 0,62 & 0,54 & 0,65 & 0,57 & 0,38 & 0,61 & 0,57 & 1,00 & & & & & & & & & \\
\hline$P 2.2 .1$ & 0,01 & 0,13 & 0,05 & $-0,05$ & $-0,03$ & 0,06 & 0,07 & 0,02 & 1,00 & & & & & & & & \\
\hline P2.2.2 & 0,07 & 0,19 & 0,13 & 0,03 & $-0,02$ & 0,10 & 0,16 & 0,06 & 0,74 & 1,00 & & & & & & & \\
\hline$P 2.2 .4$ & 0,16 & 0,24 & 0,16 & 0,14 & 0,03 & 0,19 & 0,17 & 0,22 & 0,56 & 0,46 & 1,00 & & & & & & \\
\hline$P 2.2 .5$ & 0,31 & 0,40 & 0,27 & 0,22 & 0,18 & 0,29 & 0,26 & 0,29 & 0,45 & 0,44 & 0,42 & 1,00 & & & & & \\
\hline$P 3.1$ & 0,33 & 0,23 & 0,25 & 0,20 & 0,23 & 0,30 & 0,29 & 0,29 & $-0,07$ & $-0,12$ & 0,07 & 0,01 & 1,00 & & & & \\
\hline P3.2 & 0,37 & 0,34 & 0,31 & 0,21 & 0,28 & 0,28 & 0,32 & 0,27 & $-0,06$ & $-0,03$ & 0,04 & 0,08 & 0,36 & 1,00 & & & \\
\hline P3.3 & 0,30 & 0,26 & 0,29 & 0,31 & 0,29 & 0,27 & 0,34 & 0,28 & $-0,06$ & $-0,02$ & 0,12 & 0,05 & 0,38 & 0,31 & 1,00 & & \\
\hline P3.4 & 0,29 & 0,17 & 0,32 & 0,21 & 0,26 & 0,27 & 0,31 & 0,30 & $-0,08$ & $-0,05$ & 0,09 & $-0,06$ & 0,47 & 0,42 & 0,45 & 1,00 & \\
\hline P3.5 & 0,30 & 0,24 & 0,28 & 0,13 & 0,27 & 0,23 & 0,31 & 0,22 & 0,01 & $-0,01$ & 0,09 & 0,03 & 0,57 & 0,48 & 0,43 & 0,45 & 1,00 \\
\hline
\end{tabular}

Fuente: Elaboración propia. 


\begin{tabular}{|c|c|c|}
\hline Cód. & Var. & Frase - Enunciado \\
\hline P2.1.1 & RSE_Sus1 & $\begin{array}{l}\text { Pienso que mi organización se interesa por la RSE porque realmente se preocupa por la } \\
\text { comunidad en la que opera. }\end{array}$ \\
\hline $\mathrm{P} 2.1 .2$ & RSE_Sus2 & $\begin{array}{l}\text { Mi organización se interesa en las cuestiones propias de la RSE porque se preocupa por } \\
\text { aquellos actores con los cuales interactúa y/o hace negocios. }\end{array}$ \\
\hline P2.1.3 & RSE_Sus3 & $\begin{array}{l}\text { Mi organización se interesa por programas y actividades de RSE porque quiere ayudar a } \\
\text { resolver los problemas de la comunidad. }\end{array}$ \\
\hline $\mathrm{P} 2.1 .4$ & RSE_Sus4 & $\begin{array}{l}\text { Siento que mi organización tiene un genuino interés en el bienestar de aquellos indivi- } \\
\text { duos externos que se ven afectados por su operación o negocio. }\end{array}$ \\
\hline $\mathrm{P} 2.1 .5$ & RSE_Sus5 & Mi organización considera importante ayudar a aquellos que lo necesitan. \\
\hline P2.1.6 & RSE_Sus6 & $\begin{array}{l}\text { Mi organización se involucra en programas de RSE porque realmente desea ayudar a } \\
\text { aquellos actores con los cuales interactúa y/o hace negocios. }\end{array}$ \\
\hline P2.1.7 & RSE_Sus7 & $\begin{array}{l}\text { Considero que el hecho de tener la oportunidad de interactuar con la comunidad es algo } \\
\text { que en mi compañía es altamente valorado. }\end{array}$ \\
\hline P2.1.8 & RSE_Sus8 & $\begin{array}{l}\text { Pienso que a mi organización le atrae el concepto de la RSE porque suele asumir los } \\
\text { problemas de la comunidad y de los individuos externos como si fueran propios. }\end{array}$ \\
\hline P2.2.1 & RSE_Sim1 & $\begin{array}{l}\text { Creo que mi organización se interesa por la RSE para evitar "verse mal" en frente de los } \\
\text { demás. }\end{array}$ \\
\hline $\mathrm{P} 2.2 .2$ & RSE_Sim2 & $\begin{array}{l}\text { Pienso que mi organización usa el concepto de la RSE para verse bien con respecto a sus } \\
\text { competidores y/o compañías similares. }\end{array}$ \\
\hline $\mathrm{P} 2.2 .3$ & RSE_Sim3 & $\begin{array}{l}\text { Creo que mi organización aprovecha el concepto de la RSE cuando cumple requisitos } \\
\text { legales y/o normativas de certificación (pago de impuestos, regalías, certificaciones ISO, } \\
\text { Great Place to Work, manejo de residuos, etc.) }\end{array}$ \\
\hline $\mathrm{P} 2.2 .4$ & RSE_Sim4 & $\begin{array}{l}\text { Uno de los intereses que tiene mi organización al simpatizar con la RSE, es evitar la } \\
\text { crítica (mala prensa) que sobre ella hacen algunos medios de comunicación o los actores } \\
\text { con los cuales interactúa y/o hace negocios. }\end{array}$ \\
\hline $\mathrm{P} 2.2 .5$ & RSE_Sim5 & $\begin{array}{l}\text { Creo que la organización de la cual hago parte aprovecha la RSE para parecer una com- } \\
\text { pañía ética ante la sociedad. }\end{array}$ \\
\hline $\mathrm{P} 2.2 .6$ & RSE_Sim6 & $\begin{array}{l}\text { Mi organización adopta la RSE con el objetivo de darle a entender a sus empleados que } \\
\text { existe preocupación por aquellos actores externos que de una u otra forma se relacionan } \\
\text { con su operación. }\end{array}$ \\
\hline P3.1 & OID1 & $\begin{array}{l}\text { Si alguien menciona algo negativo sobre mi organización, es como si me estuviera insul- } \\
\text { tando a mí mismo. }\end{array}$ \\
\hline P3.2 & OID2 & A mí me preocupa lo que los demás piensan acerca de mi organización. \\
\hline P3.3 & OID3 & $\begin{array}{l}\text { Cuando yo me refiero a la organización de la que soy miembro, prefiero hablar de "noso- } \\
\text { tros" en lugar que de "ellos". }\end{array}$ \\
\hline P3.4 & OID4 & El éxito de mi organización es mi propio éxito. \\
\hline P3.5 & OID5 & $\begin{array}{l}\text { Cuando alguien dice algo positivo acerca de mi organización, lo tomo casi como un } \\
\text { cumplido personal. }\end{array}$ \\
\hline P3.6 & OID6 & $\begin{array}{l}\text { Si viese alguna noticia verdadera en donde se critique o se mencione algo negativo sobre } \\
\text { mi organización, me sentiría avergonzado. }\end{array}$ \\
\hline
\end{tabular}

